\title{
Operations and Maintenance for Multipurpose Offshore Platforms using Statistical Weather Window Analysis
}

\author{
Taemin Heo*, Phong T. T. Nguyen ${ }^{\dagger}$, Lance Manuel ${ }^{*}$, Maurizio Collu $^{\ddagger}$, K A Abhinav ${ }^{\ddagger}, \mathrm{Xue}_{\mathrm{Xu}^{\ddagger}}$ and Giulio Brizzi ${ }^{\S}$ \\ *Department of Civil, Architectural and Environmental Engineering, University of Texas at Austin, Austin, Texas 78712 \\ Email: taemin@utexas.edu \\ ${ }^{\dagger}$ Faculty of Civil Engineering, HCMC University of Technology and Education, Ho Chi Minh City, Vietnam \\ Email: phongnguyen@utexas.edu \\ ${ }_{\ddagger}^{\ddagger}$ Department of Naval Architecture, Ocean \& Marine Engineering, University of Strathclyde, Glasgow, UK G4 0LZ \\ Email: maurizio.collu@strath.ac.uk \\ ${ }^{\S}$ Chlamys s.r.l., Trani, Italy \\ Email: chlamys@tiscali.it
}

\begin{abstract}
With increasing offshore-related commerce, the choice of appropriate operations and maintenance activities must take into consideration safety, costs and performance targets. Stochastic weather conditions at each site of interest presents uncertain situations. We present an optimized decisionmaking procedure that seeks to maximize monetary benefits while minimizing safety risks. Our proposed approach outlines and illustrates application of such a policy by incorporating traditional weather window analysis using a Markov Decision Process approach. In particular, the approach is applied in case study involving the operation of a multipurpose platform at an offshore Scotland site.
\end{abstract}

\section{INTRODUCTION}

Weather conditions-e.g., wind speeds, wave heights, etc.- can vary greatly over the year at offshore sites. Offshore activities associated with operations and maintenance (O\&M) of multipurpose platforms (MPPs), see Fig. 1, for an example, are gaining in importance and often need different service activities periodically and/or under constraints to avoid downtime and loss of revenue (as with energy generation from wind, waves, etc.). The literature contains examples for dealing with O\&M using weather windows in statistical analyses-e.g., in aquaculture farming, offshore wind energy generation, etc. These studies feature offshore sites around the world including the Gulf of Mexico, the east and west coasts of Japan, the west coast of Ireland, the Barents Sea, the North Atlantic Ocean, and the UK [1]-[6]. Reference [7] presented a methodology for weather window prediction that considered offshore vessels and challenges associated with operations in Norway. Reference [8] evaluated the marine environment at a Korean wind farm to select appropriate O\&M vessels.

One of the key driving factors for the long-term economical viability of MPPs, as for offshore renewable energy devices, is accessibility: the ability to keep, for example, all renewable energy systems, aquaculture systems, etc. operating. For offshore wind farms, accessibility is usually achieved through a mix of crew transfer vessels (CTVs), service operation vessels (SOVs), and helicopters that transport specialized technicians. The feasibility of an O\&M activity is assessed by comparing measured wave heights and wind speeds against operational capabilities of these transport systems. Differently, in the UK-China project, INNO-MPP (https://www.ukchn-core.com/ project/inno-mpp/), where the feasibility of a wind-aquaculture MPP at the same Scottish location discussed in this work is considered [9], [10], it has emerged that the O\&M of aquaculture systems must usually rely on technicians who will decide about the acceptability of the metocean conditions based on more intuitive and human-readable metrics such as the Beaufort scale, and not on, say, the measured significant wave height, $H_{s}$. The O\&M strategies adopted for such MPPs, then, have to take into account such human factors that can have a substantial impact on adopted policy.

With multipurpose platforms that are becoming increasingly common in the "blue economy," there is a need to be able to deal with a wide-ranging set of operations related to the intended multiple uses [11]. An archived history of past weather conditions at a selected site provides us with weather window statistics that can aid in a rational plan for O\&M. Yet, a daily optimized decision-making guide, for not only minimizing down time and loss of revenue at the facility but also for maximizing the safety of the operators, has not been systematically studied. In the literature, some good examples of studies have been undertaken that involve the development of O\&M decision models in wind energy applications. For instance, References [12]-[14] employed a Partially Observed Markov Decision Process (POMDP) to construct a stochastic model for quantifying risks and uncertainties, and developed an O\&M decision model for land-based wind turbines. Reference [15] reviewed many O\&M decision support research studies for offshore wind farms. A reliability-based computational model was also implemented to establish O\&M procedures for an 


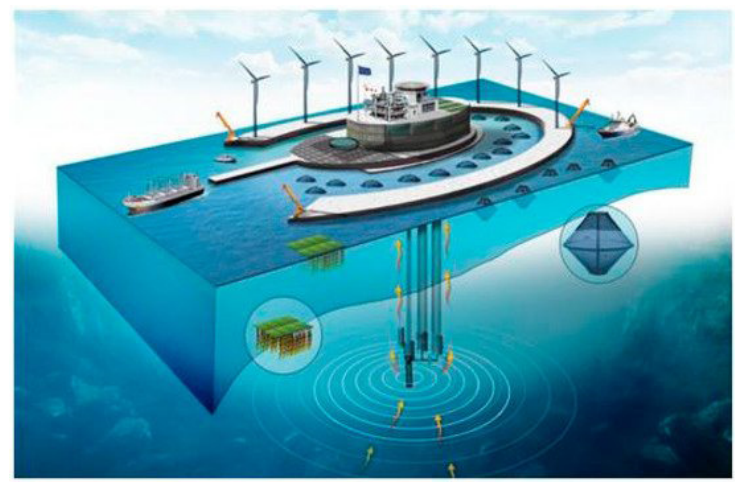

Fig. 1. Multipurpose Platform Concept [17].

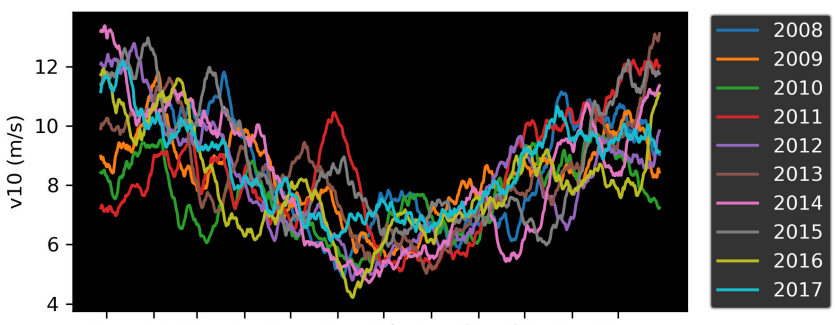

Jan Feb Mar Apr May Jun Jul Aug Sep Oct Nov Dec

Fig. 2. Temporal trends in mean wind speed at $10 \mathrm{~m}$

offshore renewable energy farm [16].

We propose, by using wind data from a Scottish site, how a Markov Decision Process can be defined that facilitates rational decision-making while taking into consideration O\&M constraints as well as site-specific stochastic weather characteristics and the safety.

\section{Materials And Methods}

\section{A. Offshore Wind Speed Data}

As far as weather conditions, this study considers the mean wind speed at $10 \mathrm{~m}\left(v_{10}\right)$ obtained every 6 hours, from 2008 to 2017 , at the location of a planned multipurpose platform offshore Scotland (lat. $56.5^{\circ}$, lon. $-7^{\circ}$ ). Fig. 2 shows the $v_{10}$ data, smoothed using a 30-day moving window for each year separately. Seasonal patterns are clear; greater variability and more severe weather is evident in the winter. These stochastic characteristics of the weather serve to highlight the challenges associated with dealing with it, while attempting to schedule and optimize O\&M activities.

\section{B. Beaufort Scale}

For the weather window analysis, $v_{10}$ can be related to the Beaufort scale, which is an empirical measure relating wind speed to observed conditions at sea and is widely used in navigation and voyage forecasts. The original scale had 13 classes (going from 0 to 12 ), but this study uses only levels 0 to 9 ; levels greater than 9 are extreme observations and account for only a small fraction of the data. Table 1 shows the original Beaufort scale and the modified version used in this study.
TABLE I

BEAUFORT WIND SCALE

\begin{tabular}{c|c|c}
\hline $\begin{array}{c}\text { Beaufort } \\
\text { Number }\end{array}$ & $\begin{array}{c}\text { Wind Speed }(\mathrm{m} / \mathrm{s}) \\
\text {-Original- }\end{array}$ & $\begin{array}{c}\text { Wind Speed }(\mathrm{m} / \mathrm{s}) \\
\text {-This Study- }\end{array}$ \\
\hline 0 & $<0.5$ & $<0.5$ \\
1 & $0.5-1.5$ & $0.5-1.5$ \\
2 & $1.5-3.3$ & $1.5-3.3$ \\
3 & $3.3-5.5$ & $3.3-5.5$ \\
4 & $5.5-7.9$ & $5.5-7.9$ \\
5 & $7.9-10.7$ & $7.9-10.7$ \\
6 & $10.7-13.8$ & $10.7-13.8$ \\
7 & $13.8-17.1$ & $13.8-17.1$ \\
8 & $17.1-20.7$ & $17.1-20.7$ \\
9 & $20.7-24.4$ & $\geq 20.7$ \\
10 & $24.4-28.4$ & \\
11 & $28.4-32.6$ & \\
12 & $\geq 32.6$ & \\
\hline
\end{tabular}

\section{O\&M Decision Making Problem Setting}

We present next the problem formulation that addresses O\&M decision making for offshore multipurpose platforms. There are different required work durations, $t_{w}$, for the different types of O\&M activities involved. Based on the O\&M vessels/equipment needed and the type of O\&M activity, operable (acceptable) weather conditions are defined in terms of maximum allowable Beaufort number, $\xi_{B}$. Then, from the data, we need to develop a time series, $X_{e}(t)$, which represents favorable time segments with operable weather conditions. Fig. 3 shows a illustrative example for an activity with $\xi_{B}=4$ in January 2008. Triangular pulse-shaped time segments increase while the Beaufort number is below the threshold, $\xi_{B}$, and go to zero whenever the weather conditions get more severe and exceed the threshold, $\xi_{B}$. When an O\&M activity needs to occurs at $t_{0}$, revenue starts to be lost after some time, $t_{a u x}$, associated with some auxiliary system spending capacity. The per unit time loss of revenue is defined as $c_{\text {down }}$; then, the loss at time $t$ is $c_{\text {down }} * \max \left(D(t)-t_{\text {aux }}, 0\right)$, where $D(t)$ is the down time (unavailable window) at time, $t$. If $X_{e}\left(t_{0}+t_{w}\right)=X_{e}\left(t_{0}\right)+t_{w}$, the O\&M activity can be successfully performed and the down time is reset to zero; otherwise, $X_{e}\left(t_{0}+t_{w}\right)-X_{e}\left(t_{0}\right)$ is less than $t_{w}$, and $D\left(t_{0}+t_{w}\right)=D\left(t_{0}\right)+t_{w}$ indicating that the O\&M activity was unsuccessful. In the latter case, a unit failure cost, $c_{f a i l}$, is charged. Successful and failed scenarios of an O\&M activity are illustrated in Fig. 3 with green and red shaded windows, respectively. Possible changes of $X_{e}(t)$ when $X_{e}\left(t_{0}\right)=i$ and $t_{w}=3$ ( $\equiv 18$ hours) are shown in Fig. 4 .

\section{Season Identification}

Fig. 2 provided an indication of seasonal variability in offshore weather at the selected site. For simplicity, a stationary 

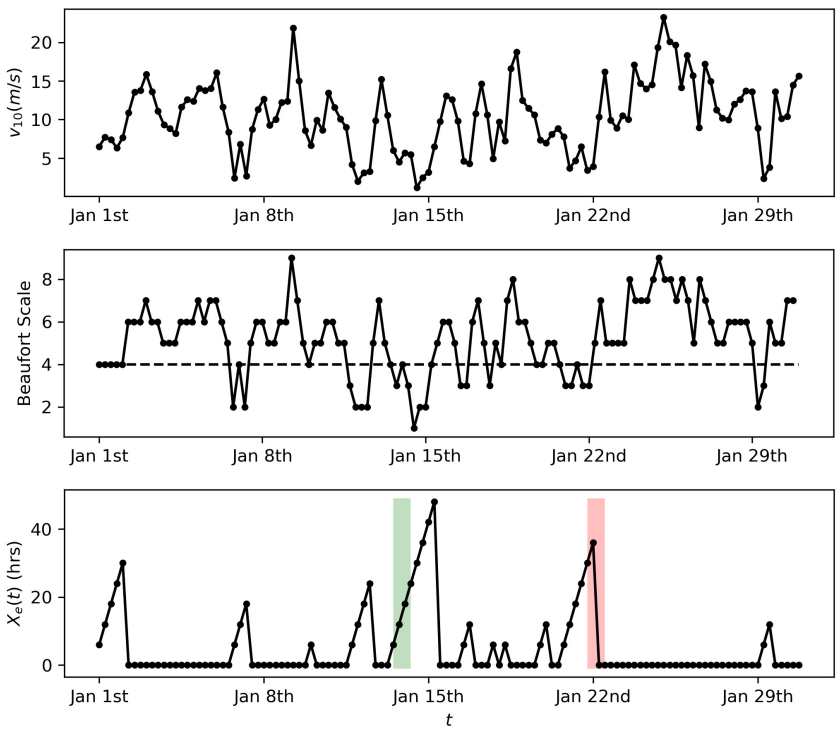

Fig. 3. Example series of $v_{10}$, Beaufort scale, and favorable times of operable weather, $X_{e}(t)$, where $\xi_{B}=4$ with illustration of successful and failed scenarios of an O\&M activity where $t_{w}=3$ ( $\equiv 18$ hours)

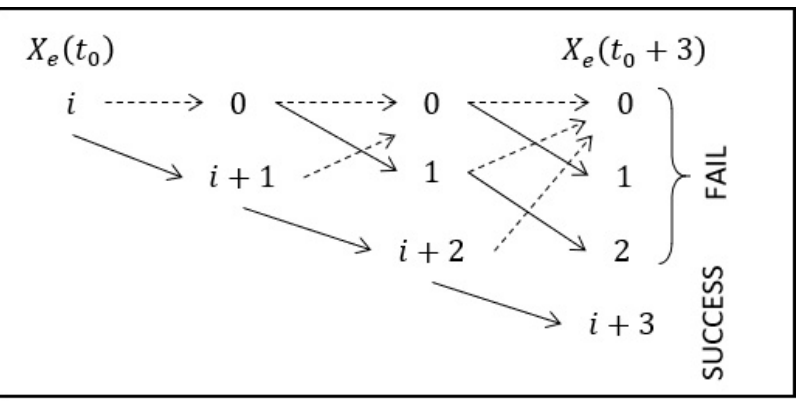

Fig. 4. Possible cases of evolution of $X_{e}$ where $t_{w}=3$ (i.e., required work duration $=18$ hours)

stochastic weather process is assumed where each year is divided into two distinct periods. We are interested in the transition of the Beaufort number with time; accordingly, three variables, $\left(B(t-1), B(t), i_{\text {time }}\right)$, are used to define seasons by k-means clustering. $B(t)$ is the Beaufort number at time $t$ while $i_{\text {time }}$ is an annual 6-hour basis time index. Three clusters with different colors are evident in Fig. 5. The transition time index can be inferred distinctly for each seasons as the clusters do not overlap. The purple cluster has benign conditions (low Beaufort numbers), and its time index range is that of the summer season, ranging from $490(=122.5$ days $=$ May 3 rd $)$ to 976 (= 244 days $=$ Sep 1st $)$.

\section{E. Markov Decision Process}

A Markov Decision Process (MDP) is a discrete time stochastic control process that can model situations where outcomes are partly random and partly under the control of a decision maker. An MDP is a 4-tuple $(S, A, P, R)$ of state, action, transition probability and cost (or reward). Reward is common nomenclature in defining a MDP but, in the present

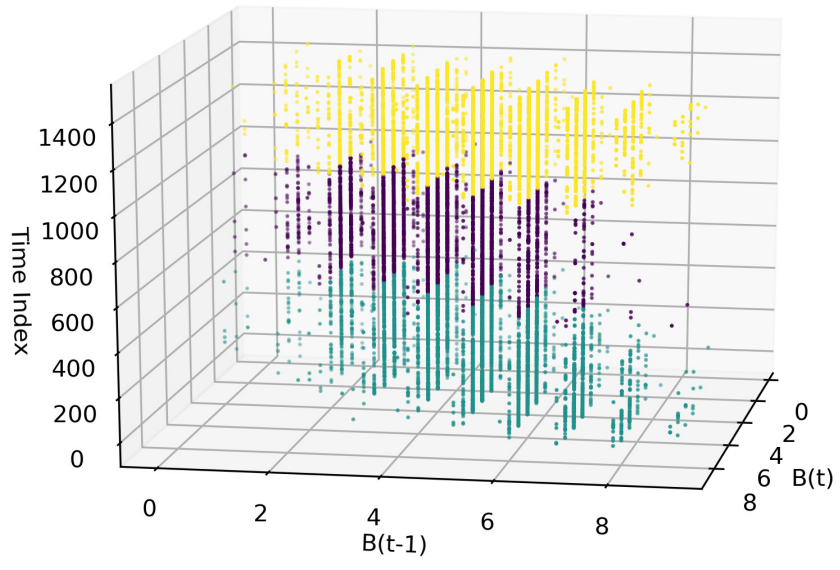

Fig. 5. Season identification result

study, it is more appropriate to discuss cost. At each discrete time, the process is in state $s \in S$, while the decision maker takes action $a \in A$ and incurs cost $R(s, a)$ as a result of the action. The process switches to a new state $s^{\prime}$ according to a known stochastic model, with transition probability $P\left(s^{\prime} \mid s, a\right)$. The state transitions of an MDP satisfy the classical Markov property:

$$
P\left(s_{t+1} \mid s_{t}, a_{t}, s_{t-1}, a_{t-1}, \ldots, s_{0}, a_{0}\right)=P\left(s_{t+1} \mid s_{t}, a_{t}\right) .
$$

Then, the MDP helps to yield an optimized policy $\pi^{*}(s)$. The policy maps states to actions and the optimal policy is iteratively found by exploring the best action for each state that minimizes the overall cost function. Here, a discount factor $\gamma$, $0 \leq \gamma \leq 1$, is introduced to account for present and future value of relevant item. Dynamic programming, introduced by Bellman (1954), may be used to solve the MDP [18]. In this study, to provide general guidelines that are independent of time, we assume an infinite horizon problem and the value iteration is formulated as:

$$
\begin{array}{r}
V^{\pi^{*}}(s)=\max _{a \in A}\left[\sum_{s^{\prime} \in S} P\left(s^{\prime} \mid s, a\right) R\left(s, a, s^{\prime}\right)+\right. \\
\\
\left.\gamma \sum_{s^{\prime} \in S} P\left(s^{\prime} \mid s, a\right) V^{\pi^{*}}\left(s^{\prime}\right)\right]
\end{array}
$$

where $V^{\pi}$ is a value function that represents the expected overall cost of policy $\pi$. The iterations terminate when the difference between the value function in two consecutive steps is below a specified level $\varepsilon$ (more details can be found in [19]).

\section{F. Markov Decision Process Setting for the Problem}

In the present study, there are only two actions, "Go" and "Stay". The data are time-indexed every 6 hours. The range for both favorable and down times is $(0, \infty)$. In the finite discrete MDP process setting that we adopt, we limit this range for favorable and down times. The maximum favorable and down times are limited to 126 hours and 54 hours, respectively. For convenience, a selected number of intervals is used to indicate 
favorable and down times. As a result, the favorable times are $\{0,1,2, \ldots, 21\}$ and down times are $\{0,1,2, \ldots, 9\}$. Every possible pair of favorable and down times is considered a state; 220 states are defined accordingly. An $n^{\text {th }}$ state indicates $(n$ mod 21) time steps of favorable time and a quotient $(n / 21)$ time steps of down time. Transition probabilities are defined using a matrix of dimension, $2 \times 220 \times 220$ (resulting from $220 \times 220$ inter-state transition probabilities and two possible actions at each state). Finally, the cost function is also defined using a matrix of dimension, $220 \times 2$ (resulting from costs incurred by the two actions for the 220 states).

The transition probability matrix is constructed from the data in two steps. First, an intermediate transition matrix of favorable times is developed from $X_{e}$. A transition event from $X_{e}(t)=i$ to $X_{e}\left(t+t_{w}\right)=i^{\prime}$ (where $i^{\prime} \in\left\{0,1, \ldots, t_{w}-\right.$ $\left.\left.1, i+t_{w}\right\}\right)$ is monitored and each occurrence is counted and included in the matrix of dimension, $22 \times 22$. By normalizing this count-based matrix row by row, the intermediate transition matrix results. Second, a reshaping of the matrix considering actions and the down time, $D(t)$, to yield the final transition probability matrix for the MDP is needed. When the action is "Stay", $D(t)$ will transition to $D(t)+t_{w}$, regardless of $X_{e}(t)$ until $D(t)+t_{w}>9$ is true, since the consequence of the action "Stay" is independent of the stochastic weather process and we limit down time to 9 time steps (54 hours). When $D(t)+t_{w}>9$, the down time is assumed to stay at 9. The action "Go" results in two scenarios. When the O\&M activity is successful-i.e., $X_{e}\left(t+t_{w}\right)=X_{e}(t)+t_{w}$ and $D(t+$ $\left.t_{w}\right) \rightarrow 0$. If there was at least one bad weather-i.e., $X_{e}(t+$ $\left.t_{w}\right)<t_{w}$, then the O\&M activity is assumed to have failed and $D\left(t+t_{w}\right)=D(t)+t_{w}$. Algorithms 1 and 2 in Fig. 6 indicate the two steps in the transition probability matrix construction procedure; Fig. 7 shows the structure of the transition matrix.

A cost matrix is constructed next. For each action "Stay", $c_{\text {down }}$ is charged for every state that has longer down time than $t_{\text {aux }}$. For each action "Go", $c_{\text {down }}$ is also charged for every state that has longer down time than $t_{a u x}$. Additionally, $c_{\text {fail }}$ is charged for states with more severe weather than $\xi_{B}$. For states that indicate successful O\&M activity-i.e., zero down time-no cost is charged. Algorithm 3 in Fig. 6 shows how the cost matrix is constructed while Fig. 7 shows the structure of the cost matrix.

\section{A CASE STUdy}

A case study with $\xi_{B}=5, t_{w}=4, t_{\text {aux }}=2, c_{\text {down }}=-1.5$, and $c_{\text {fail }}=-1$ is considered and the constructed transition matrices are shown in Fig. 8. Note that $t_{a u x}=2$ means that for the O\&M activity of interest, a loss of revenue results after 2 time steps (12 hours) of unfavorable weather; a duration of 4 time steps (24 hours) of favorable weather with Beaufort number not greater than 5 is needed for this O\&M activity. The cost associated with lack of use (down time) is 1.5 times higher than the cost of failure. As a result of the value iteration,
Algorithm 1. Constructing intermediate transition matrix

$$
\begin{aligned}
& \text { 1: input } X_{e}, t_{w} \\
& \text { 2: for all } t \text { where } X_{e}(t)>21 \text { do } X_{e}(t) \leftarrow 21 \\
& \text { 3: generate a zero matrix } \mathbf{A}_{22 \times \mathbf{2 2}} \\
& \text { 4: for } i=0,1, \ldots, 21 \text { do } \\
& \text { 5: } \mathbf{A}[i, 0] \quad=\frac{\sum \mathbf{1}_{X_{e}(t)=i n X_{e}\left(t+t_{w}\right)=0}}{\sum \mathbf{1}_{X_{e}(t)=i}} \\
& \text { 6: } \mathbf{A}[i, i+1]=\frac{\sum \mathbf{1}_{X_{e}(t)=i n X_{e}\left(t+t_{w}\right)=i+1}}{\sum \mathbf{1}_{X_{e}(t)=i}}
\end{aligned}
$$

Algorithm 2. Constructing final transition probability matrix

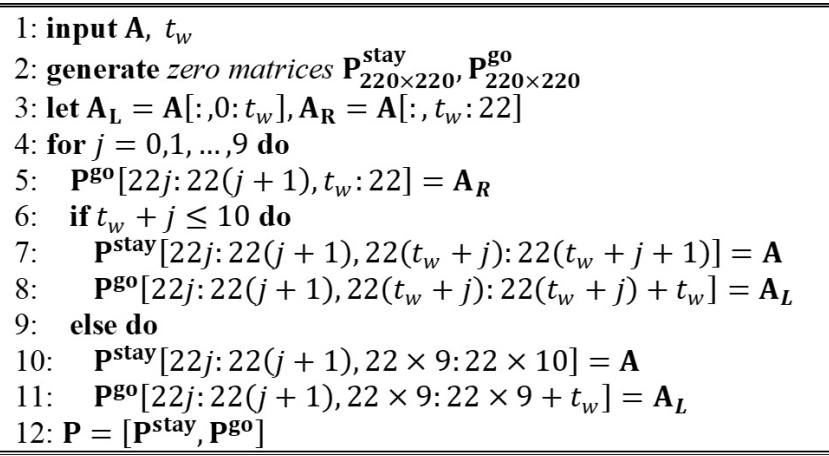

Algorithm 3. Constructing cost matrix

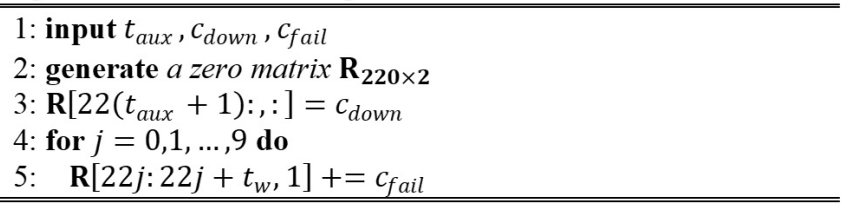

Fig. 6. Algorithms for constructing transition probability matrix and cost matrix

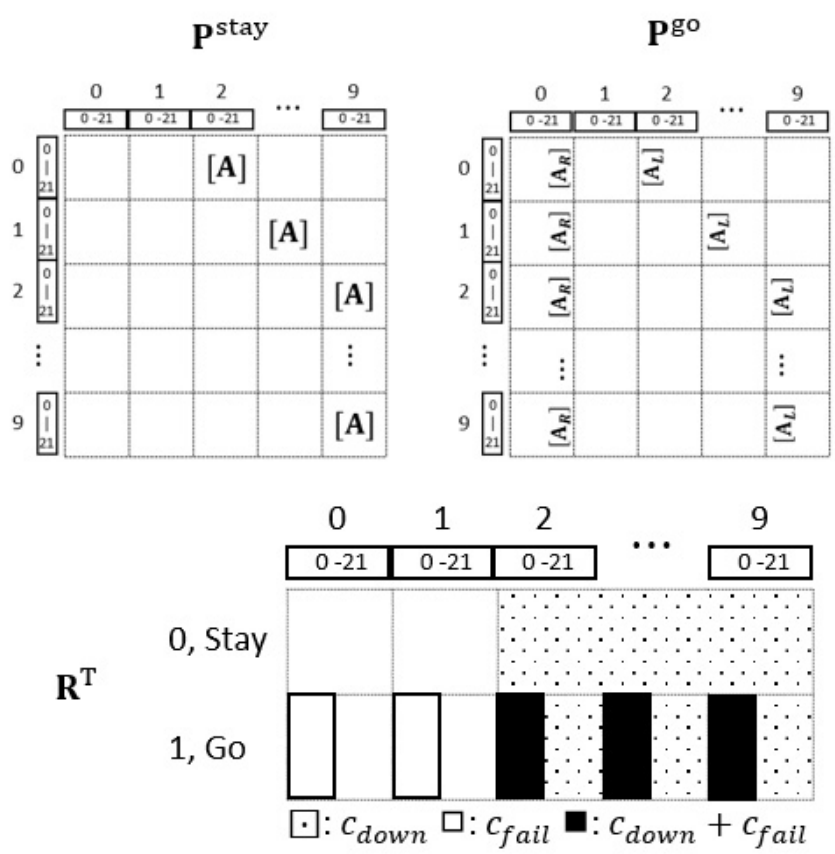

Fig. 7. Structure of the transition matrix and the cost matrix when $t_{w}=2$ ( $\equiv 12$ hours), $t_{\text {aux }}=1$ ( $\equiv 6$ hours) 

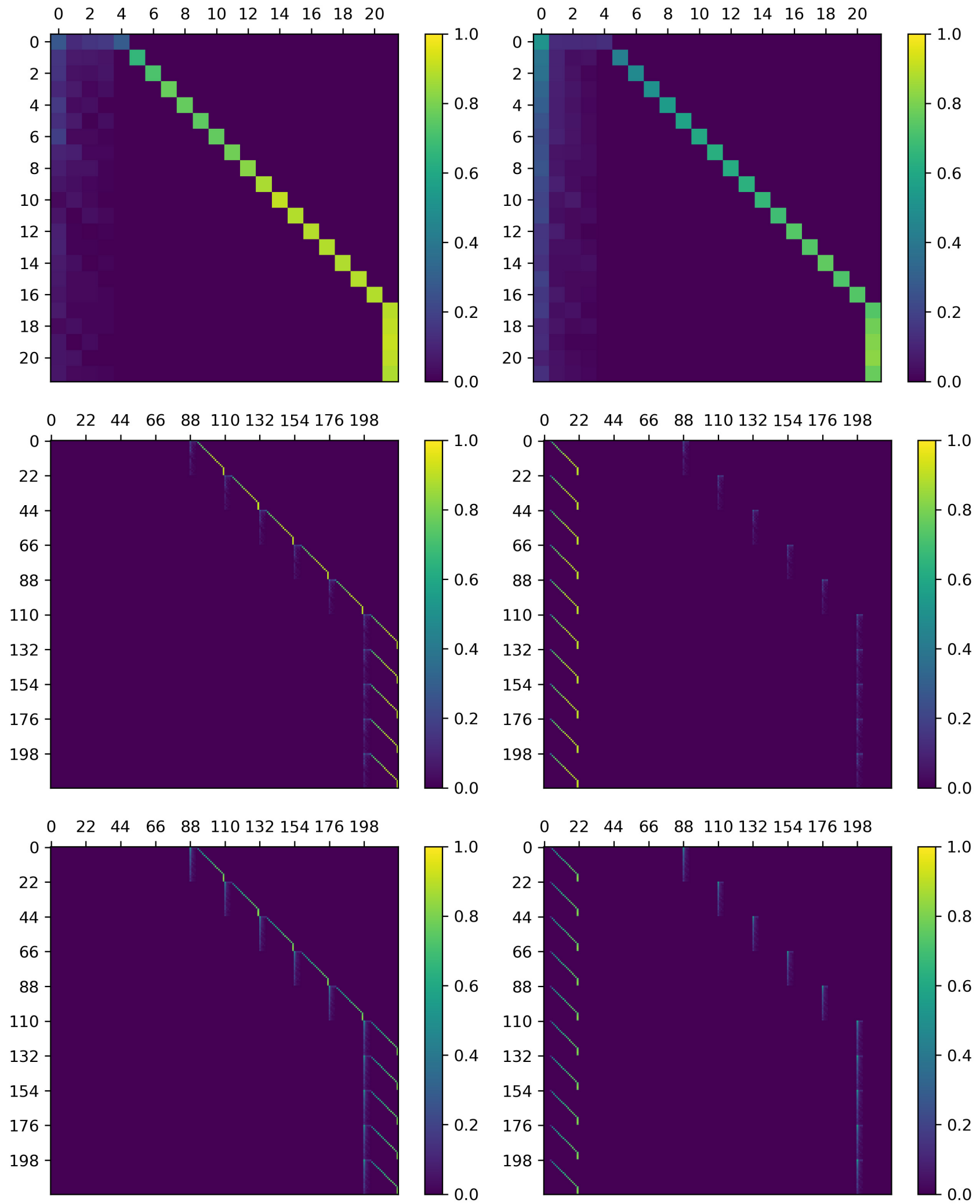

Fig. 8. Constructed intermediate transition matrix $\mathbf{A}_{\text {summer }}$ (upper left), $\mathbf{A}_{\text {winter }}$ (upper right), final transition matrix elements $\mathbf{P}_{\text {summer }}^{\text {stay }}$ (center left), $\mathbf{P}_{\text {summer }}^{\text {go }}$ (center right), $\mathbf{P}_{\text {winter }}^{\text {stay }}\left(\right.$ lower left), $\mathbf{P}_{\text {winter }}^{\text {go }}$ (lower right). 
the policy is optimized as follows:

$$
\begin{gathered}
\pi_{\text {summer }}^{*}(s)= \begin{cases}0, & (s \bmod 21) \leq 1 \\
1, & \text { Otherwise }\end{cases} \\
\pi_{\text {winter }}^{*}(s)= \begin{cases}0, & (s \bmod 21) \leq 3 \\
1, & \text { Otherwise }\end{cases}
\end{gathered}
$$

From the results, one can infer that, in the summer, a duration of favorable time of operable weather longer than 2 time steps (12 hours) indicates a "Go" action to do the O\&M activity is recommended. In the more severe weather conditions and greater variability in the stochastic weather process in the winter, a duration of favorable time of operable weather longer than 4 time steps ( 24 hours) is needed to recommend "Go". This optimized policy is independent of the down time since $c_{\text {down }}$ is assumed constant; however, loss of revenue varies linearly with down time. In some situations, the loss in revenue may be exponential with down time or, as for a fish farm, $c_{\text {down }}$ may be a function of down time; then, an optimized policy will be dependent on down time. In another scenario, it is possible that favorable weather is indicated and its duration exceeds 21 time steps; then, a "Go" action is recommended at the site due to highly stationary weather.

\section{CONCLUSION}

This study proposed a framework to develop an O\&M strategy for multipurpose offshore platforms. The approach describes an optimized decision making policy given stochastic weather data, as well as O\&M activity and cost constraints. Exhaustive evaluation of all scenarios is possible using a Markov Decision Policy framework that can be employed in planned blue economy projects.

\section{ACKNOWLEDGMENT}

The 4th, 5th, and 6th authors are supported by the UK Engineering and Physical Sciences Research Council UK (EPSRC) and the Natural Environment Research Council UK (NERC), through Grants EP/R007497/1 and EP/R007497/2, and the Natural Science Foundation of China (NSFC) through Grant 51761135013.

\section{REFERENCES}

[1] M. Du, J. Yi, P. Mazidi, L. Cheng, and J. Guo, "An approach for evaluating the influence of accessibility on offshore wind power generation," Preprints, 2017.

[2] Y. Kikuchi and T. Ishihara, "Assessment of weather downtime for the construction of offshore wind farm by using wind and wave simulations," in Journal of Physics: Conference Series, vol. 753, 2016, p. 092016.

[3] M. O'Connor, T. Lewis, and G. Dalton, "Weather window analysis of irish west coast wave data with relevance to operations \& maintenance of marine renewables," Renewable energy, vol. 52, pp. 57-66, 2013.

[4] A. Orimolade and O. Gudmestad, "On weather limitations for safe marine operations in the barents sea," in IOP Conf. Ser. Mater. Sci. Eng, vol. 276, 2017, p. 012018.

[5] D. Martins, G. Muraleedharan, and C. Guedes Soares, "Analysis on weather windows defined by significant wave height and wind speed," Renewable Energies Offshore, vol. 91, 2015.

[6] J. Paterson, F. D’Amico, P. Thies, R. Kurt, and G. Harrison, "Offshore wind installation vessels-a comparative assessment for uk offshore rounds 1 and 2," Ocean Engineering, vol. 148, pp. 637-649, 2018.
[7] T. Gintautas and J. D. Sørensen, "Improved methodology of weather window prediction for offshore operations based on probabilities of operation failure," Journal of Marine Science and Engineering, vol. 5, no. 2, p. 20, 2017.

[8] D. Ahn, S.-C. Shin, S.-Y. Kim, H. Kharoufi, and H.-C. Kim, "Comparative evaluation of different offshore wind turbine installation vessels for korean west-south wind farm," International Journal of Naval Architecture and Ocean Engineering, vol. 9, no. 1, pp. 45-54, 2017.

[9] K. Abhinav, M. Collu, S. Ke, and Z. Binzhen, "Frequency domain analysis of a hybrid aquaculture-wind turbine offshore floating system," in 37th International Conference on Ocean, Offshore and Arctic Engineering, ASME, Glasgow, Scotland, June 2019.

[10] K. A. Abhinav, X. Xu, Z. Lin, and M. Collu, "Dynamic response of a multi-purpose floating offshore structure under extreme sea conditions," in International Conference on Ships and Offshore Structures ICSOS 2020, Glasgow, Scotland, September 2020.

[11] M. Vierros and C. De Fontaubert, "The potential of the blue economy: increasing long-term benefits of the sustainable use of marine resources for small island developing states and coastal least developed countries," The World Bank, Tech. Rep., 2017.

[12] E. Byon, L. Ntaimo, and Y. Ding, "Optimal maintenance strategies for wind turbine systems under stochastic weather conditions," IEEE Transactions on Reliability, vol. 59, no. 2, pp. 393-404, 2010.

[13] E. Byon and Y. Ding, "Season-dependent condition-based maintenance for a wind turbine using a partially observed markov decision process," IEEE Transactions on Power Systems, vol. 25, no. 4, pp. 1823-1834, 2010.

[14] E. Byon, "Wind turbine operations and maintenance: a tractable approximation of dynamic decision making," IIE Transactions, vol. 45, no. 11, pp. 1188-1201, 2013.

[15] H. Seyr and M. Muskulus, "Decision support models for operations and maintenance for offshore wind farms: A review,' Applied Sciences, vol. 9, no. 2, p. 278, 2019.

[16] G. Rinaldi, P. Thies, R. Walker, and L. Johanning, "A decision support model to optimise the operation and maintenance strategies of an offshore renewable energy farm," Ocean Engineering, vol. 145, pp. 250262, 2017.

[17] Y.-T. Sie, P.-A. Château, Y.-C. Chang, and S.-Y. Lu, "Stakeholders opinions on multi-use deep water offshore platform in Hsiao-Liu-Chiu, taiwan," International Journal of Environmental Research and Public Health, vol. 15, no. 2, p. 281, 2018.

[18] R. Bellman, "The theory of dynamic programming," Rand Corp. Santa Monica CA, Tech. Rep., 1954.

[19] K. G. Papakonstantinou and M. Shinozuka, "Planning structural inspection and maintenance policies via dynamic programming and markov processes. Part I: Theory," Reliability Engineering \& System Safety, vol. 130, pp. 202-213, 2014. 